\title{
Under the genomic radar: The Stealth model of Alu amplification
}

\author{
Kyudong Han, ${ }^{1}$ Jinchuan Xing, ${ }^{1}$ Hui Wang, Dale J. Hedges, Randall K. Garber, \\ Richard Cordaux, and Mark A. Batzer ${ }^{2}$ \\ Department of Biological Sciences, Biological Computation and Visualization Center, Center for BioModular Multi-Scale Systems, \\ Louisiana State University, Baton Rouge, Louisiana 70803, USA
}

\begin{abstract}
Alu elements are the most successful SINEs (Short INterspersed Elements) in primate genomes and have reached more than 1,000,000 copies in the human genome. The amplification of most Alu elements is thought to occur through a limited number of hyperactive "master" genes that produce a high number of copies during long evolutionary periods of time. However, the existence of long-lived, low-activity Alu lineages in the human genome suggests a more complex propagation mechanism. Using both computational and wet-bench approaches, we reconstructed the evolutionary history of the AluYb lineage, one of the most active Alu lineages in the human genome. We show that the major $A l u Y b$ lineage expansion in humans is a species-specific event, as nonhuman primates possess only a handful of $A l u Y b$ elements. However, the oldest existing AluYb element resided in an orthologous position in all hominoid primate genomes examined, demonstrating that the AluYb lineage originated 18-25 million years ago. Thus, the history of the $A l u \mathrm{Yb}$ lineage is characterized by $\sim 20$ million years of retrotranspositional quiescence preceding a major expansion in the human genome within the past few million years. We suggest that the evolutionary success of the Alu family may be driven at least in part by "stealth-driver" elements that maintain low retrotranspositional activity over extended periods of time and occasionally produce short-lived hyperactive copies responsible for the formation and remarkable expansion of Alu elements within the genome.
\end{abstract}

[The sequence data from this study have been submitted to GenBank under accession nos. AY791249-AY791290.]

As the most successful SINEs (Short INterspersed Elements) in primate genomes, Alu elements have enjoyed remarkable proliferation during the primate radiation and have expanded to more than 1,000,000 copies in the human genome (Lander et al. 2001; Batzer and Deininger 2002). Despite considerable progress in the understanding of their biology and distribution throughout primate taxa (Salem et al. 2003b; Singer et al. 2003; Carter et al. 2004; Hedges et al. 2004; Otieno et al. 2004; Roos et al. 2004; Ray et al. 2005), a great deal of uncertainty still remains concerning their strategy for survival. It has been generally accepted that the amplification of most Alu elements occurs through a small number of long-lived high activity "master" genes (Deininger et al. 1992; Deininger and Batzer 1993), although there is considerable debate as to the details of this amplification strategy (Matera et al. 1990; Schmid 1993; Batzer et al. 1995; Cordaux et al. 2004; Price et al. 2004). In this model, the mutations accumulated in the master genes are inherited by the copies they produced, and consequently, a series of hierarchical Alu subfamilies that share the novel diagnostic mutation(s) are generated (Slagel et al. 1987; Willard et al. 1987; Britten et al. 1988; Deininger and Slagel 1988; Jurka and Smith 1988; Deininger et al. 1992; Batzer and Deininger 2002). On the other hand, the evolutionary history of the AluYa5 lineage, one of the most active human Alu lineages, suggests that the founder gene of this Alu lineage existed long before the major expansion of the lineage within the human genome

\footnotetext{
${ }^{1}$ These authors contributed equally to this work.

${ }^{2}$ Corresponding author.

E-mail mbatzer@Isu.edu; fax (225) 578-7113.

Article and publication are at http://www.genome.org/cgi/doi/10.1101/ gr.3492605.
}

(Leeflang et al. 1993). Contrary to the prediction of the master gene model, it has maintained low retrotranspositional activity, and this founder gene itself may not be directly responsible for the propagation of the recent human AluYa5 elements (Shaikh and Deininger 1996). Thus, these studies suggest that the expansion of $A l u$ elements may follow a more complex propagation mechanism. Unfortunately, aside from the AluYa5 lineage, little data exist concerning the evolutionary origin of other Alu subfamilies in humans, making it difficult to assess the evolutionary significance of the results reported in the original AluYa5 lineage studies.

To gain additional insight into Alu subfamily propagation, we reconstructed the evolutionary history of the Alu Yb lineage, one of the largest and most active Alu lineages in the human genome (Jurka 1993; Carter et al. 2004) that composes $~ 40 \%$ of the human-specific Alu elements (Hedges et al. 2004). This lineage, originally termed $\mathrm{Sb} 2$, is characterized by a 7-nt duplication involving positions 246 through 252 of the AluY consensus sequence (Jurka 1993; Batzer et al. 1996). The AluYb lineage in the human genome is subdivided into three major subfamilies as follows: AluYb7, AluYb8, and AluYb9 (Batzer et al. 1996; RoyEngel et al. 2001; Jurka et al. 2002; Carter et al. 2004) based on diagnostic mutations following the standardized nomenclature for Alu repeats (Batzer et al. 1996). The majority of the human Alu $\mathrm{Yb}$ elements integrated into the genome during the last 3 to 4 million years (Myr) and reached a total copy number of about 2000 elements (Carter et al. 2004). The human diseases caused by de novo AluYb8 insertions suggest that this subfamily is currently actively retrotransposing, and a comprehensive analysis of the $\mathrm{Yb}$ lineage indicated that about $20 \%$ of $A l u \mathrm{Yb}$ elements are poly- 
morphic in human genome (Muratani et al. 1991; Oldridge et al. 1999; Carter et al. 2004). Previous studies suggested that the evolutionary history of the AluYb lineage is much older than its period of major expansion in the human genome, and $A l u \mathrm{Yb}$ elements have also been identified in other nonhuman primates (Zietkiewicz et al. 1994; Gibbons et al. 2004). Nevertheless, the extent to which these elements are distributed among nonhuman primate species remains undetermined.

Using both a computational approach and polymerase chain reaction (PCR) display methodology, we have determined the distribution of $A l u \mathrm{Yb}$ elements in different hominoid genomes. We find that the long-term evolutionary history of the Alu $\mathrm{Yb}$ lineage exhibits a pattern that is remarkably similar to that of the AluYa lineage. Thus, the evolution of the AluYa and AluYb lineages illustrate a common strategy for Alu element proliferation. We propose a model for the expansion and survival of Alu elements in the primate order.

\section{Results}

\section{AluYb elements in the common chimpanzee genome}

To determine the evolutionary history of the $A l u \mathrm{Yb}$ lineage, we first computationally retrieved all of the AluYb elements from the first draft of the common chimpanzee (Pan troglodytes) genomic sequence (panTro1 Nov. 2003 assembly). A total of 12 AluYb elements were identified and subjected to PCR amplification on a common chimpanzee population panel and a separate primate panel composed of human and eight additional nonhuman primates (see Methods). The number of $A l u \mathrm{Yb}$ elements recovered from the chimpanzee draft sequence is in good agreement with a previous study (Gibbons et al. 2004). Detailed information on each locus, including primer sequences, annealing temperature, PCR product sizes, and chromosomal locations are shown in Table 1 . Among the 12 elements identified, 10 belong to the AluYb8 subfamily, while the other two are non-AluYb7/8/9 elements (Table 2). Of the $10 \mathrm{AluYb8}$ elements, four loci were spe- cific to the common chimpanzee lineage, including three elements that were polymorphic within the chimpanzee population panel (Figs. 1, 2B). Five elements were shared between the pygmy chimpanzee and common chimpanzee lineage (Fig. 2C) and one was present within human, chimpanzee, and gorilla genomes. These results suggest that the initial expansion of the AluYb8 subfamily predates the divergence of gorillas and humans/ chimpanzees, which is thought to have occurred $\sim 7$ million years ago (Mya) (Goodman et al. 1998). In addition, two nonAluYb7/ 8/9 elements displayed PCR amplicon sizes consistent with the presence of an Alu element in all of the hominoid primates we tested.

To confirm that the PCR products were derived from authentic AluYb elements, all of the filled amplicons were cloned and sequenced. This sequencing effort revealed additional insight into the evolutionary history of the following four loci: Pan1, Pan2, Pan3, and Pan4. At the Pan1 locus, an AluYb8 element was inserted into the chimpanzee lineage (pygmy and common) after the divergence of humans and chimps. However, the pygmy chimpanzee and common chimpanzee showed both filled and empty size amplicons in their PCR amplifications, suggesting the presence of an Alu insertion polymorphism in both species (Fig. 3A). Sequence analysis of the locus showed that the AluYb8 element inserted immediately upstream of an AluSx element in the same orientation, and that the integration site is partially shared (three copies of direct repeats). More recently, a nonhomologous recombination or intrachromosomal recombination event in the common chimpanzee genome generated a hybrid Alu element that is composed of the first half of the older AluSx element and the second half of the newly inserted AluYb8 element. In contrast, the smaller allele amplified in other primates appeared to be the preintegration site of the AluYb8 element (Fig. 3B).

DNA sequence analysis of the Pan2 and Pan3 loci resulted in the recovery of two gene conversion events. The Pan2 locus appears to be the oldest Alu element that we recovered. PCR analysis of the locus showed the presence of an Alu element or filled

Table 1. AluYb loci identified in this study

\begin{tabular}{|c|c|c|c|c|c|c|c|}
\hline \multirow[b]{2}{*}{ Name $^{a}$} & \multirow{2}{*}{$\begin{array}{l}\text { Forward primer } \\
\text { sequence }\left(5^{\prime}-3^{\prime}\right)\end{array}$} & \multirow{2}{*}{$\begin{array}{l}\text { Reverse primer } \\
\text { sequence }\left(5^{\prime}-3^{\prime}\right)\end{array}$} & \multirow{2}{*}{$\begin{array}{l}\text { Chr. loc. } \\
\text { in human }\end{array}$} & \multirow[b]{2}{*}{ Direct repeat } & \multirow[b]{2}{*}{ A. T. ${ }^{\mathrm{c}}$} & \multicolumn{2}{|c|}{ Product size } \\
\hline & & & & & & Filled & Empty \\
\hline Pan1* & ACCAAAATGCAGGTCTCTTGTT & ССТTTTCTTTATGCGCATTTCT & 5:171753413 & АAAACATCСТC & 55 & 892 & 561 \\
\hline Pan2* & CCTGGCCTATTGATGATTTTCT & GCCTCAGAAGGAGTTTTGTTGT & $12: 42243314$ & AAAACTCCСTCTGAG & 58.6 & 481 & 129 \\
\hline Pan3* & CCGATATCATGCATTTTCCATA & TGGCAAGAAAGACATGATTGAA & 3:155790154 & AAAAATAAATACCA & 60 & 728 & 412 \\
\hline Pan4* & AGGGCAATTACTATGTTTCAGGA & TTTTTCACGTTCTTACAATAGAACA & $18: 66254955$ & ACAATAGAACATTCCT & 55 & 403 & 52 \\
\hline Pan5* & GCTTCATTTCTGCCTGCTTATT & TCTGCAAATTTAACTCCAAACC & 10:97871078 & AAAAACAGCAAGT & 55 & 504 & 181 \\
\hline Pan6* & GGCATTTTAAGCTCTTTGATAGC & CATGCTAGACATGAGAACAAACA & $2: 183429337$ & GAAATAGTTCCTGCT & 60 & 749 & 257 \\
\hline Pan7* & GCAGCTGCTTTCTGTCTCTGTA & TCAGCAACAATAAGGAACGAAG & $3: 49401325$ & AAAAGAAACCAGTCAC & 55 & 502 & 175 \\
\hline Pan8* & CCCAGATTGATTCTTCCCTTTA & ATGCCAGTTCCATTATTTCCAC & 8:101149947 & AAGAGAGAAGAAA & 55 & 834 & 506 \\
\hline Pan9* & CCAGGACCCAGAGCTTAGTAGT & TCCAAAGGAATATGATGTCACAG & $14: 52808462$ & AAAGAAATTGATT & 55 & 434 & 111 \\
\hline Pan10* & TGTGGCAGATTTTATTGTAGACTT & TCCATGCTCTTGGAGTAAATGA & 4:72958275 & АААААААТTСАТСТG & 55 & 500 & 174 \\
\hline Pan11* & AGGAGCTCTTCTTAGAAAATCCA & AAAGATCCACGACTAGGCACAT & $2: 214354697$ & AAAGAAAAAGGAAAAAAC & 60 & 521 & 193 \\
\hline Pan12* & CGTTAGCTCTGGATTTTTCATGT & TATTCTCCACCATGACCAAGTG & X:93270745 & AAAAATTAGCCGGGCA & 60 & 871 & 548 \\
\hline Gorilla7" & TGAAGAATTGGGAGGAAAAGAA & ACTGCAAGATTGCTGTTCACAT & $13: 32666822$ & AAAAAATGACTAACAG & 55 & 583 & 261 \\
\hline Gorilla8 ${ }^{\#}$ & GCACTCATCCGTACCTGACTTA & TCAGAGCATCTCTTTCTGTCCA & 2:229645741 & AAAAAAGGAGGCAA & 60 & 540 & 219 \\
\hline Gorilla16" & AATTCTTTGGGGTAGGTGGAAT & CACAACGTACACCCTAAAATGG & $X: 24004038$ & AAGAGTTGCAGTTGCTCA & 55 & 531 & 202 \\
\hline Gorilla19\# & CAAGTTGTGTTATGTGAGGTTTTGA & GGAGCCCTAATGTATAGCATGG & 7:10262937 & AAGAGAGTAGATCTT & 59.5 & 597 & 279 \\
\hline Gorilla21" & AACAAGAGATGCTAGAAAGCCAAT & CGGAGTTGGACACATTTCTTTT & $1: 51666132$ & AAAGAAAGGAGGA & 55 & 749 & 434 \\
\hline Pygmy4 $^{\#}$ & GCAGTTTTTCCCATTTGCTCTA & TTGAGTCTTTTTCTGGGCTTTC & $2: 130058820$ & AAGACTTACTATA & 55 & 600 & 281 \\
\hline
\end{tabular}

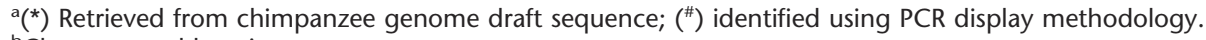

${ }^{\mathrm{b} C h r o m o s o m a l ~ l o c a t i o n . ~}$

${ }^{\mathrm{C}}$ Annealing temperature. 
Table 2. Primate phylogenetic PCR panel results for chimpanzee AluYb elements

\begin{tabular}{|c|c|c|c|c|c|c|c|c|c|}
\hline Name & $\begin{array}{c}\text { Chimpanzee } \\
\text { panel }\end{array}$ & $\begin{array}{c}\text { Human } \\
\text { (HeLa) }\end{array}$ & $\begin{array}{c}\text { Pygmy } \\
\text { chimpanzee }\end{array}$ & Gorilla & Orangutan & Gibbon & Siamang & $\begin{array}{l}\text { Green } \\
\text { monkey }\end{array}$ & $\begin{array}{c}\text { Owl } \\
\text { monkey }\end{array}$ \\
\hline Pan1 & $\begin{array}{l}\text { Fixed present } \\
\text { (AluYb8) }\end{array}$ & - & $\begin{array}{c}\text { Polymorphic } \\
\text { (AluYb8) }\end{array}$ & - & - & - & - & $x$ & $x$ \\
\hline Pan2 & $\begin{array}{l}\text { Fixed present } \\
\text { (AluYb6) }\end{array}$ & $+($ AluYb6) & $+($ AluYb6) & $+($ AluYb6) & $+($ AluYb6) & $+($ AluYb6) & $+($ AluYb6) & $+($ AluSg $)$ & $x$ \\
\hline Pan3 & $\begin{array}{l}\text { Fixed present } \\
\quad(\text { AluYb) }\end{array}$ & $+($ AluYb) & $+($ AluYb) & $+($ AluYb) & $+($ AluY $)$ & $+($ AluY $)$ & $+($ AluY $)$ & - & $x$ \\
\hline Pan4 & $\begin{array}{c}\text { Fixed present } \\
\text { (AluYb8) }\end{array}$ & $+($ AluYb8) & $+($ AluYb8) & $+($ AluYb8) & - & - & - & - & - \\
\hline Pan5 & $\begin{array}{l}\text { Polymorphic } \\
\text { (AluYb8) }\end{array}$ & - & 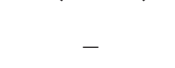 & 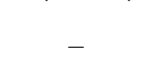 & - & - & - & - & $x$ \\
\hline Pan6 & $\begin{array}{l}\text { Fixed present } \\
\text { (AluYb8) }\end{array}$ & - & $+($ AluYb8) & - & - & - & - & - & - \\
\hline Pan7 & $\begin{array}{l}\text { Fixed present } \\
\text { (AluYb8) }\end{array}$ & - & $+($ AluYb8) & - & - & - & - & - & - \\
\hline Pan8 & $\begin{array}{c}\text { Fixed present } \\
\text { (AluYb8) } \\
\text { Fixed present }\end{array}$ & - & - & - & - & - & - & - & - \\
\hline Pan9 & $\begin{array}{l}\text { Fixed present } \\
\text { (AluYb8) }\end{array}$ & - & $+($ AluYb8) & - & - & - & - & - & - \\
\hline Pan10 & $\begin{array}{l}\text { Fixed present } \\
\text { (AluYb8) }\end{array}$ & - & $+($ AluYb8) & - & - & - & - & - & - \\
\hline Pan11 & $\begin{array}{l}\text { Polymorphic } \\
\text { (AluYb8) }\end{array}$ & - & - & - & - & - & - & - & $x$ \\
\hline Pan12 & $\begin{array}{l}\text { Polymorphic } \\
\text { (AluYb8) }\end{array}$ & - & - & - & - & - & - & - & - \\
\hline
\end{tabular}

(+/-) Presence/Absence of Alu element; (x) no amplification.

allele in all of the hominoid primates we examined. The green monkey also showed a filled size amplicon, but the amplicon was slightly smaller than the predicted size (Fig. 4A). DNA sequence analysis showed that all hominoid primates possessed an AluYb element at the Pan2 locus with the AluYb lineage diagnostic duplication and five of the eight additional mutations that charac-

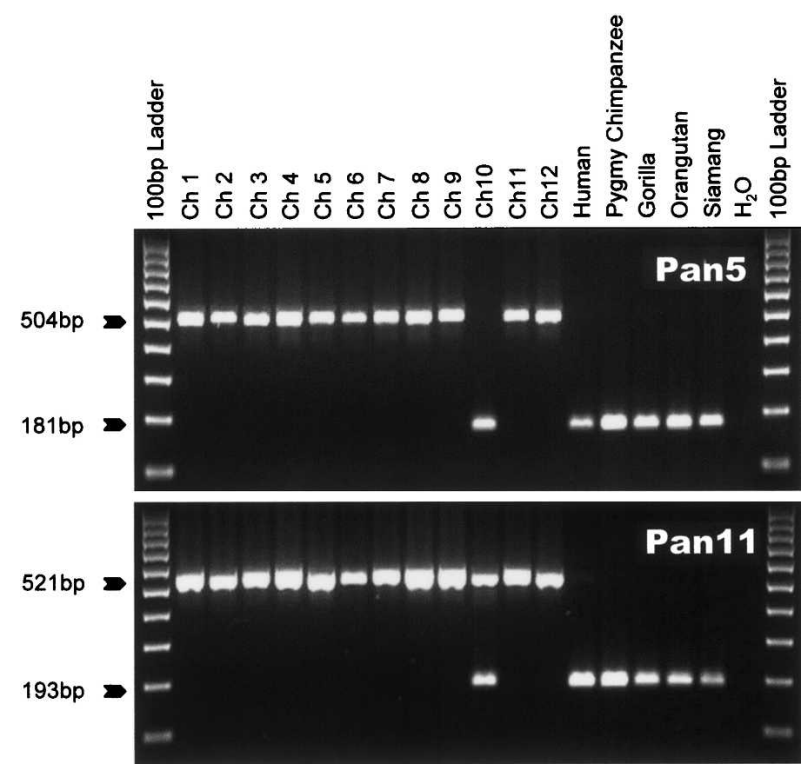

Figure 1. Polymorphic AluYb8 elements in the common chimpanzee genome. Gel chromatographs of two loci are shown. The locus name is shown at right. The product sizes for filled and empty alleles (preintegration size) are indicated at left. The DNA panel is composed of 12 unrelated common chimpanzee individuals and other primate species. The template used in each reaction is listed at top. terize the Alu Yb8 consensus sequence. However, an AluSg element was found in the orthologous locus in green monkey. This result suggests that the AluYb6 element (missing two diagnostic mutations compared with the AluYb8 consensus) at the Pan2 locus was introduced into the genome via a gene conversion event after the divergence of Old World monkeys and hominoids, but before the radiation of the hominoid primates. Thus, the AluYb6 element at the Pan2 locus is 18-25 Myr old (Goodman et al. 1998). Given the sequence identity between the AluYb and the AluSg consensus sequences, we estimate that the starting point of the gene conversion event was located within the first 75 bp of the Alu element, and that the 3' terminus was located between positions \#267 and \#310.

The Pan3 locus also contains a gene conversion event (Fig. 4B). Siamang, gibbon, and orangutan possess an Alu element that does not contain the AluYb lineage diagnostic duplication and has the highest sequence identity to the AluY consensus. However, the Alu elements in the gorilla, chimpanzee, and human genomes belong to the $A l u \mathrm{Yb}$ lineage, as indicated by the presence of the $A l u \mathrm{Yb}$ diagnostic duplication in these elements. This indicates that a gene conversion event occurred after the divergence of gorillas and orangutans, but prior to the divergence of gorillas, humans, and chimpanzees. During this process, a small proportion of the ancestral AluY element has been converted to an AluYb-like sequence, including the AluYb diagnostic duplication and another adjacent tightly linked diagnostic mutation. The gene-converted region could be as small as 8 bp (251-259) or as long as $30 \mathrm{bp}(237-267)$ in this case. Thus, the overall gene conversion rate (2/12) we observed here seems to be much higher than in previous studies (Maeda et al. 1988; Kass et al. 1995; Roy-Engel et al. 2002; Salem et al. 2003a). However, this is not surprising, since the difference may be due to the small sample size in the current study or reflect the longer evolutionary time period that each element may have been subjected to gene conversion. 
A)

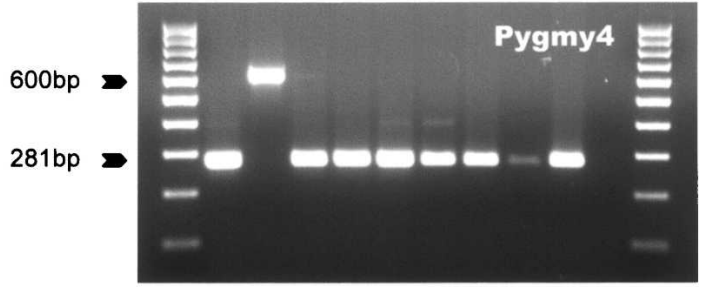

B)

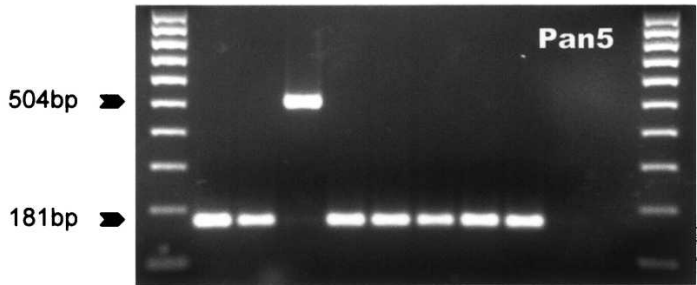

C)

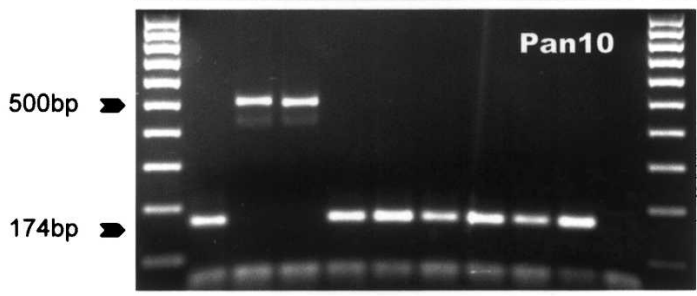

D)

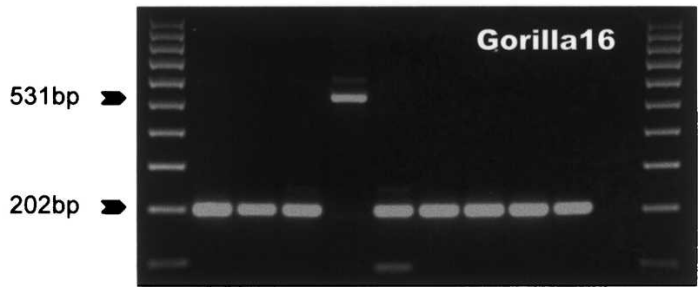

Figure 2. Lineage-specific AluYb8 insertions. Four examples of gel chromatographs are shown. The locus designation is shown at right, while the product sizes for filled and empty alleles (pre-integration size) are indicated at left. The DNA template used in each reaction is listed at top. (A) Pygmy chimpanzee-specific AluYb8 insertion. (B) Common chimpanzee-specific AluYb8 insertion. (C) Chimpanzee lineage-specific AluYb8 insertion. (D) Gorilla-specific AluYb8 insertion.

The Pan4 locus contains the oldest AluYb8 element we identified. Interestingly, the Pan 4 AluYb8 element in the human genome only has one CpG mutation $(\mathrm{G} \rightarrow \mathrm{A})$ at position \#5, compared with the AluYb8 consensus sequence, while the chimpanzee and gorilla AluYb8 elements at the orthologous Pan4 locus each have accumulated five species-specific mutations compared with the AluYb8 consensus sequence. Since no mutations are shared by the human, chimpanzee, and gorilla AluYb8 elements at the Pan4 locus, we believe that the ancestral AluYb8 element at the Pan4 locus was a canonical AluYb8 element. Using the BLAT program (http://genome.ucsc.edu/cgi-bin/hgBlat?command= start), we identified three additional AluYb8 elements in the human genome with 100\% sequence identity to the Pan 4 element. This result suggests that the Pan4 element may still be retrotranspositionally active in the human genome, although we cannot completely rule out the possibility that these four elements independently mutated at position \#5. Multiple alignments of the Pan1, Pan2, Pan3, and Pan4 loci are available on our Web site under publications (http://batzerlab.lsu.edu).

\section{AluYb insertions in other primate genomes}

To further investigate the propagation of the Alu Yb lineage, we next searched for the presence of $A l u \mathrm{Yb}$ elements in additional nonhuman primate genomes. Since no complete draft genomic sequences are available other than human and common chimpanzee, we used a modified PCR display method to identify AluYb elements from other primate genomes (see Methods). The display procedure was performed for four hominoid primates: pygmy chimpanzee, gorilla, orangutan, and gibbon; three Old World monkeys: green monkey, rhesus monkey, and pig-tailed macaque. In addition, this approach was also applied to the common chimpanzee to identify additional AluYb elements absent from the common chimpanzee genomic sequence. Two restriction enzymes were used for every template, and a minimum of 72 colonies were sequenced for each species. For the common chimpanzee, a total of seven $A l u \mathrm{Yb}$ elements were retrieved, all of which had previously been identified from the draft sequence. This suggested that our method involving two restriction enzymes yielded a $\sim 60 \%$ coverage (7/12) of the Alu Yb elements in the genome. One pygmy chimpanzee-specific and five gorillaspecific AluYb8 insertions were identified and confirmed by a PCR analysis of nonhuman primates (Fig. 2A,D) and DNA sequencing. No AluYb element was recovered from the orangutan genome using the PCR display approach. The only locus identified within the gibbon genome was Pan2, which had been previously identified in the chimpanzee genome using the computational approach. Despite at least two trials for each species, no Alu Yb elements were identified in the three Old World monkeys (green monkey, rhesus monkey, and pig-tailed macaque) examined. All of the new Alu Yb loci identified by PCR display are listed in Table 2.

Age estimates for the AluYb8 insertions in chimpanzee and gorilla

To estimate the average age of AluYb8 elements in the chimpanzee genome, CpG and non-CpG mutation densities were calculated for 10 chimpanzee-specific AluYb8 elements as reported previously (Xing et al. 2004) using the chimpanzee AluYb8 consensus. The 10 elements contained a total of nine non-CpG mutations of 2420 nucleotides and $10 \mathrm{CpG}$ mutations of $460 \mathrm{CpG}$ nucleotides. The mutation densities were $0.37 \% \pm 0.30 \%$ (average \pm standard deviation) and $2.17 \% \pm 2.29 \%$ for the non-CpG nucleotides and CpG nucleotides, respectively. Using a neutral mutation rate of $0.0015 /$ site/Myr for non-CpG sites and a mutation rate of $0.0090 /$ site/Myr for CpG sites (Xing et al. 2004), the average non-CpG and CpG mutation densities yield age estimates of $2.48 \pm 2.03$ and $2.42 \pm 2.55 \mathrm{Myr}$, respectively. For the gorilla-specific AluYb8 elements, a total of five elements were analyzed. The mutation densities were $0.17 \% \pm 0.23 \%$ and $3.48 \% \pm 3.30 \%$ for the non-CpG and CpG sites, yielding age estimates of $1.10 \pm 1.51$ and $3.86 \pm 3.66 \mathrm{Myr}$, respectively.

Phylogenetic analysis of $A l u Y b$ elements in the human genome The presence of $A l u \mathrm{Yb} 8$ elements in different primate genomes suggests that the origin of the AluYb8 subfamily may be much older than its major expansion in humans. In addition, the pres- 
A

B

Pygmy chimpanzee Common chimpanzee

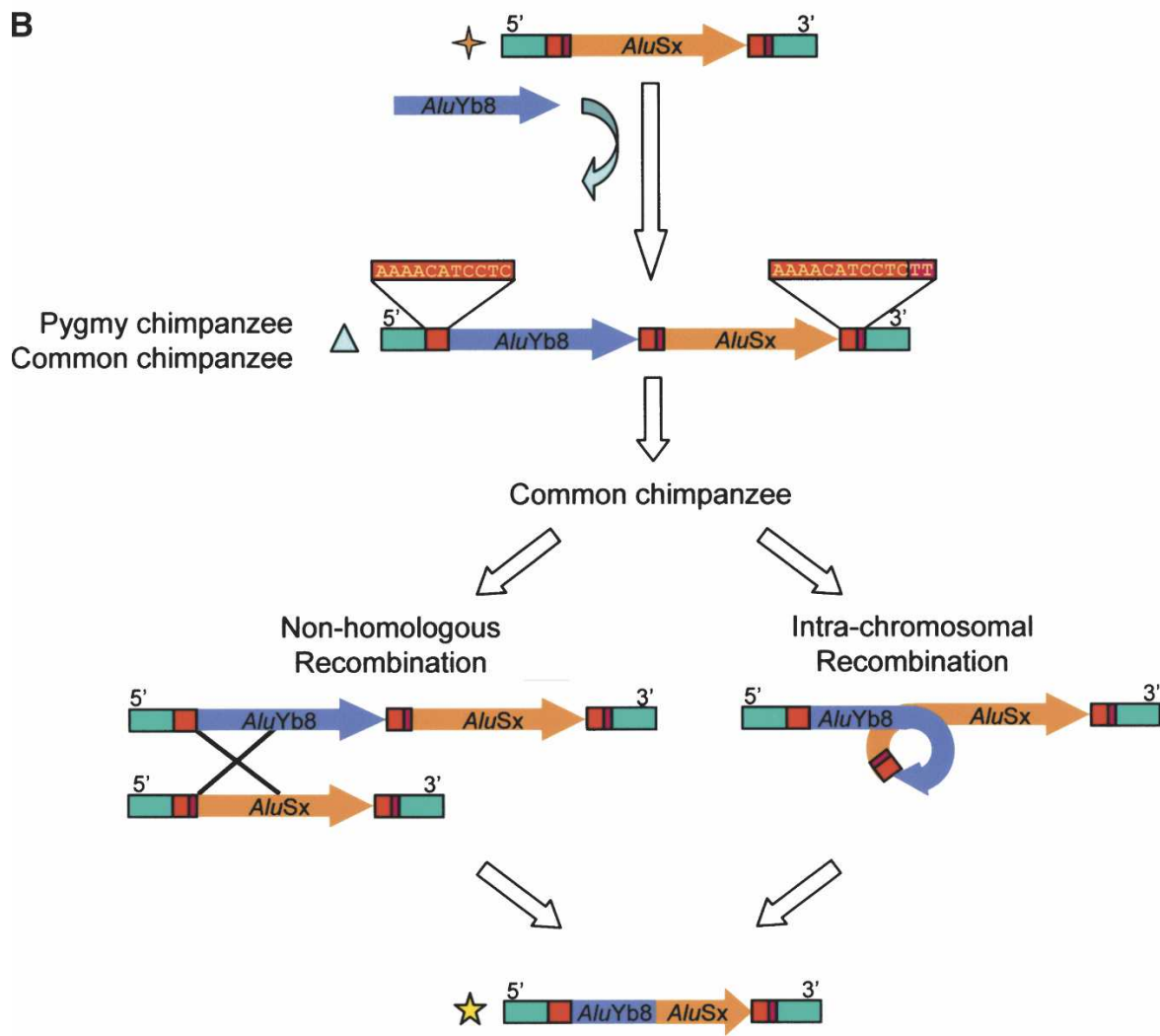

3

Figure 3. Sequence analysis of the Pan 1 locus. $(A)$ The gel chromatographs of PCR amplification results are shown. The template used in each lane is listed at the top of the gel. The product sizes for filled and empty sites (pre-integration size) are indicated at left. (B) Schematic diagrams for the possible evolutionary scenarios. Light-blue triangles denote the amplicons with an AluYb8 insertion; orange crosses denote the pre-integration products, and the yellow star denotes the recombination product in the common chimpanzee genome. Flanking sequences are shown as green boxes; target site direct repeats are shown in red and pink boxes. Alu elements are shown as arrows and the direction of arrow indicates the orientation $\left(5^{\prime} \rightarrow 3^{\prime}\right)$, with the head of the arrow denoting the end of the Alu elements.

ence of AluYb8, but not AluYb7 or AluYb9 elements in the chimpanzee genome suggests that the AluYb8 subfamily may be the ancestral component of the human AluYb lineage and would, therefore, predate the other two major human AluYb subfamilies (i.e., AluYb7 and AluYb9). To test this hypothesis, we examined the phylogenetic relationships of the different components of the Alu Yb lineage in the human genome, using a median-joining network approach (Cordaux et al. 2004) as implemented in the software NETWORK 4.1 (Bandelt et al. 1999) available at http://www.fluxusengineering.com/sharenet.htm. From the $A l u \mathrm{Yb}$ elements identified by Carter et al. (2004) in the human genome, we removed truncated elements and members of the $A l u \mathrm{Yb} 7, A l u \mathrm{Yb} 8$, and $A l u \mathrm{Yb} 9$ subfamilies, leaving 36 previously unclassified $A l u \mathrm{Yb}$ elements. After deleting the middle A-rich region and poly-A tail of the elements, a network of the Alu Yb lineage was reconstructed using the 36 non- $A l u \mathrm{Yb} 7 / 8 / 9$ elements and the consensus sequences of the $A l u \mathrm{Yb} 7$, AluYb8, and AluYb9 subfamilies. Collectively, these three subfamilies comprise $\sim 2000$ copies. A preliminary analysis suggested that several nucleotide positions may have mutated more than once. Thus, the $A l u \mathrm{Yb}$ network was calculated with these putative hypervariable positions down-weighted to 1 (positions \#64, \#98, and \#144) or 5 (position \#174 and position \#211), while other positions were given a weight of 10 . The resulting network (Fig. 5) shows that the AluYb8 subfamily occupies a central position in the network. In addition, the AluYb8 node is associated with the highest number of direct branches (nine), as compared with the AluYb7 and AluYb9 nodes (four branches each). Finally, $72 \%$ of the nonAluYb7/8/9 elements are more closely related to $A l u \mathrm{Yb} 8$ than to $A l u \mathrm{Yb} 7$ or AluYb9. Taken together, these results are strongly suggestive that the AluYb8 elements are ancestral to the other $A l u \mathrm{Yb}$ subfamilies in the human genome (Posada and Crandall 2001; Cordaux et al. 2004).

\section{Discussion}

\section{The origin of the AluYb lineage}

The AluYb lineage is one of the most active Alu lineages in the human genome, with an estimated copy number of 2000 (Carter et al. 2004). To obtain further insight into the origin of the $A l u \mathrm{Yb}$ lineage in the primate order, we analyzed the draft sequence of the common chimpanzee genome and identified 12 Alu $\mathrm{Yb}$ insertions, 10 of which are members of the AluYb8 subfamily, while the other two are non-Yb7/ $8 / 9$ elements. The presence of an AluYb element at the Pan2 locus within siamang and gibbon genomes suggested the Alu Yb lineage originated before the divergence of all hominoid primates. However, no AluYb elements have been identified in multiple oldworld monkey genomes; thus, the origin of the AluYb lineage was after the divergence of Old World monkeys and apes. These results place the origin of the AluYb lineage at the early stage of the 
A)
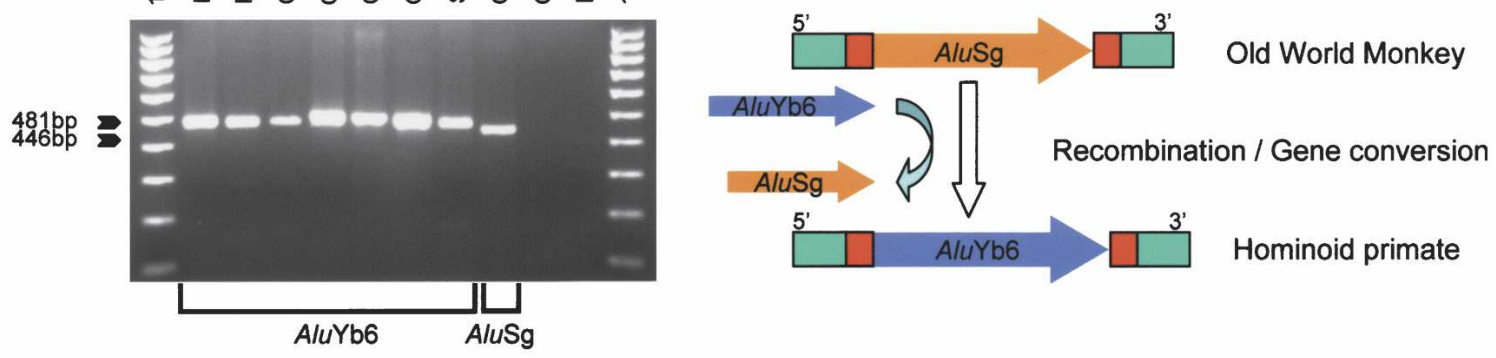

B)
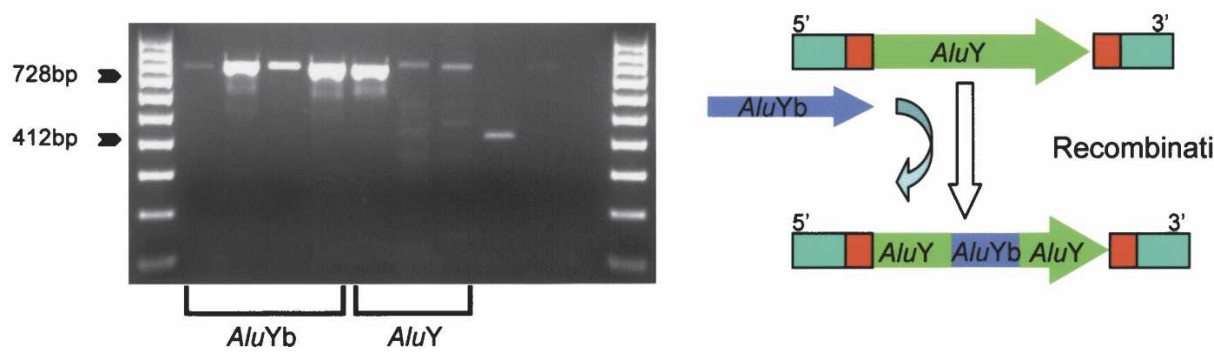

Siamang, Gibbon and Orangutan

Figure 4. Gene conversion of AluYb elements. Gel chromatographs of PCR products derived from a phylogenetic analysis of the Pan2 locus $(A)$ and Pan3 locus $(B)$ are shown at left. The DNA template used in each lane is shown at top. The product sizes for filled and empty alleles (pre-integration size) are indicated at left. The schematic diagrams depict the potential evolutionary scenarios on the right. Flanking sequences are shown as green boxes; target site direct repeats are shown in red boxes. Alu elements are shown as arrows, and the direction of the arrow indicates the orientation $\left(5^{\prime} \rightarrow 3^{\prime}\right)$, with the head of the arrow denoting the end of the Alu elements.

hominoid evolution, about 18-25 Mya (Goodman et al. 1998) It is worth noting that although the AluYb6 insertion at the Pan2 locus is the oldest $A l u Y b$ element we identified, it was most likely generated via a gene conversion event. Therefore, it may not be the founder gene of the $A l u \mathrm{Yb}$ lineage, but rather an early offspring of the Alu Yb founder gene, which was subsequently lost in extant primates.

The Pan4 AluYb element appears to have been fixed in the human, chimpanzee, and gorilla genomes, and contains all eight diagnostic mutations that characterize the AluYb8 consensus sequence. This suggests that within the $10 \mathrm{Myr}$ or so after the $A l u \mathrm{Yb}$ lineage initially arose, it was not very active in terms of retrotransposition, if at all. However, this lineage retained its retrotranspositional potential during this extended period of time. Furthermore, our results suggest that in gorilla and chimpanzee genomes, the copy number of AluYb elements is two orders of magnitude lower than that in human. Therefore, over several million years following the insertion of the AluYb8 at the Pan4 locus, the $A l u \mathrm{Yb}$ lineage still retained a very low retrotransposition activity ( 1.5 fixed copies/Myr in the chimpanzee genome) until the major expansion of the $A l u \mathrm{Yb}$ lineage in the human genome within the past 3-4 Myr (Carter et al. 2004; Xing et al. 2004).

\section{The "stealth driver" model of Alu evolution}

The long evolutionary history of AluYb lineage leads to the conclusion that the AluYb lineage has remained in the genome with little or no retrotransposition activity for an extended period of time, while retaining the ability to generate an appreciable num- ber of new copies later in a species-specific manner (Fig. 6). This scenario is different from the classic "master" gene model, in which a master gene is defined as an element that is highly active over long periods of time (Deininger et al. 1992). In general, the amplification dynamics of the AluYb lineage show a striking similarity to that of the AluYa5 lineage (Leeflang et al. 1993; Shaikh and Deininger 1996). Although the existence of lowactivity Alu source genes has previously been suggested for the AluYa5 subfamily (Shaikh and Deininger 1996), here we provide evidence that low retrotransposition activity Alu source genes should be recognized as a major factor driving Alu expansion and evolution.

We propose a model of Alu evolution and retrotransposition, in which the low-activity Alu elements are termed "stealth drivers." In contrast to master genes, stealth drivers are not responsible for generating the majority of new Alu copies, but rather for maintaining genomic retrotransposition capacity over extended periods of time. By generating new Alu copies at a slow rate, a stealth driver may generate some daughter elements that are capable of much higher retrotransposition rates. These hyperactive daughter elements may act as master genes for the amplification of Alu subfamilies and are responsible for producing the majority of the subfamily members. In addition, the new master genes may also give birth to additional retrotranspositionally active Alu copies that serve as secondary master genes or sprouts and also contribute to the expansion of the subfamily (Deininger and Batzer 1993). This view is supported by the fact that recently integrated human Alu subfamilies typically contain $\sim 15 \%$ of such secondary master genes, in addition to the primary 


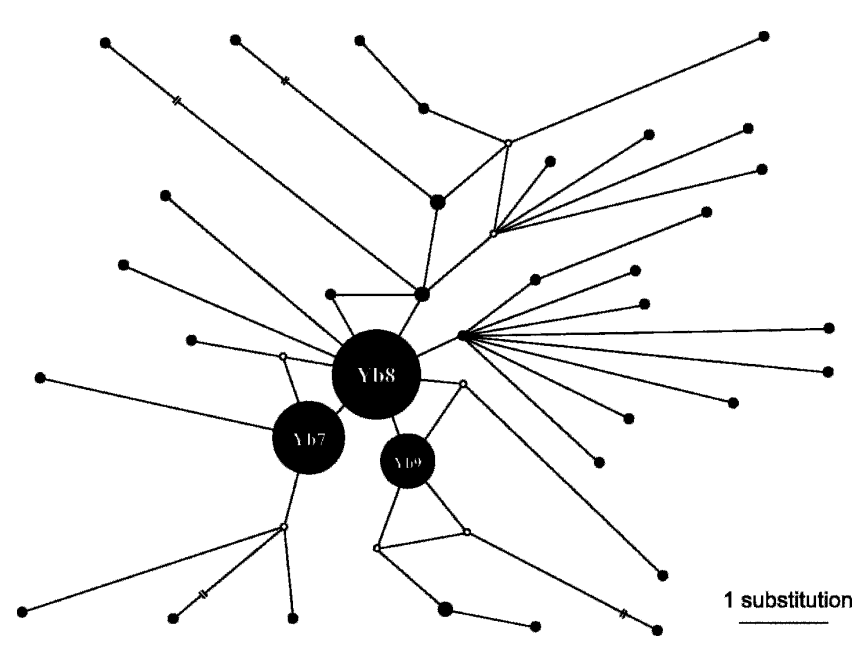

Figure 5. Median-joining network of the human-specific AluYb elements. The network of the AluYb lineage was reconstructed using the 36 non-Yb7/8/9 elements and the consensus sequences of the $\mathrm{Yb} 7, \mathrm{Yb} 8$, and Yb9 subfamilies as representatives of these three subfamilies. Black circles denote sequence types. Reconstructed nodes are identified as empty circles. The size of circles indicates the number of Alu loci with this sequence type, while arbitrary sizes were chosen for the $\mathrm{Yb} 7 / 8 / 9$ nodes to represent the relative sizes of the three subfamilies. Lines denote substitution steps, with a one-step distance being indicated in the bottom-right corner. Broken lines indicate that the length of the branch is not drawn to scale.

subfamily master gene (Cordaux et al. 2004). While the highly active master genes that are produced from the stealth driver would be deleterious and generally be subject to negative selection, the low-activity stealth driver itself will not be subject to such selection and would allow the lineage to persist for extended periods of time. Furthermore, both stealth driver and its daughter elements will also generate new elements that exhibit low levels of retrotransposition activity, effectively becoming new stealth-driver elements. In fact, it is possible that the continuation of a lineage over extended periods of time may depend on the production of new driver elements.

But the question remains as to why, after persisting relatively quietly for millions of years, a lineage can show a sudden increase in numbers, as appears to be the case for AluYa and $A l u \mathrm{Yb}$ lineages. Under the stealth-driver model, the master or stealth locus does not need to be "turned on," as one or more such drivers has been active during the entire history of the lineage. There are multiple scenarios that may account for the sudden expansion of the Alu lineage. In the scenario that we favor, periods of rapid expansion may be related to the ability of highly active daughter elements to escape selection at the population level and, consequently, produce more progeny. The change in the efficiency of natural selection in weeding out overactive elements may be related to population bottlenecks or other demographic factors (Hedges et al. 2004). Alternatively, periodic increases in element numbers may simply be due to the stochastic nature by which active daughter elements are produced. Yet another possibility is that, contingent on the relative abundance of available L1 retrotransposition machinery, there may be fluctuation in Alu expansion rates (Dewannieux et al. 2003). Less likely is the possibility that molecular host defense mechanisms, which were previously suppressing the activity of Alu elements, failed for some reason during these periods.

In the $A l u \mathrm{Yb}$ lineage, multiple lines of evidence suggest that the AluYb8 element at the Pan4 locus may be a recent stealthdriver gene in human-specific Alu retrotransposition. First, the Pan4 element is the oldest AluYb8 element we identified; second, it only accumulated one point mutation over the last $7 \mathrm{Myr}$; third, the presence of three identical human AluYb8 elements is consistent with recent low levels of retrotransposition of this element. On the other hand, since the Pan 4 AluYb8 element has accumulated five mutations in the chimpanzee and gorilla genome, and there are no other AluYb elements in these genomes with the same mutations, the AluYb8 element at Pan4 locus is unlikely to be the current driver gene in the chimpanzee and gorilla genomes. The reasons why the Pan 4 AluYb8 element may be a stealth driver in human, but not chimpanzee and gorilla, are unclear. However, there is a striking correlation between sequence similarity to the AluYb8 consensus sequence and total number of $A l u \mathrm{Yb}$ copies in these species, raising the possibility of mutational inactivation of the Pan 4 AluYb8 element in chimpanzee and gorilla, but not in the human lineage.

Previous studies have shown that the amplification of the Alu family reached its peak about 30 Mya and subsequently underwent retrotranspositional quiescence (Shen et al. 1991; Britten 1994). Although we still do not know the underlying mechanisms for the retrotranspositional quiescence, the stealth-driver model may explain why the Alu lineage has been subjected to periods of retrotranspositional quiescence interspersed with episodic bursts of amplification, as suggested by the accumulation of at least 5000 human-specific Alu elements since the humanchimpanzee divergence (Carroll et al. 2001; Batzer and Deininger 2002; Xing et al. 2003; Carter et al. 2004; Otieno et al. 2004). The AluYa and AluYb lineages that comprise $>60 \%$ of the humanspecific Alu elements may be just two successful examples of this strategy. Similar patterns of amplification have also been observed in the retrotransposition of rodent SINE family, ID, (Kim et al. 1994) and the rodent LINE (Long INterspersed Element) family, Lx (Pascale et al. 1993). In addition to these evolutionary observations, there is also experimental evidence that indicates

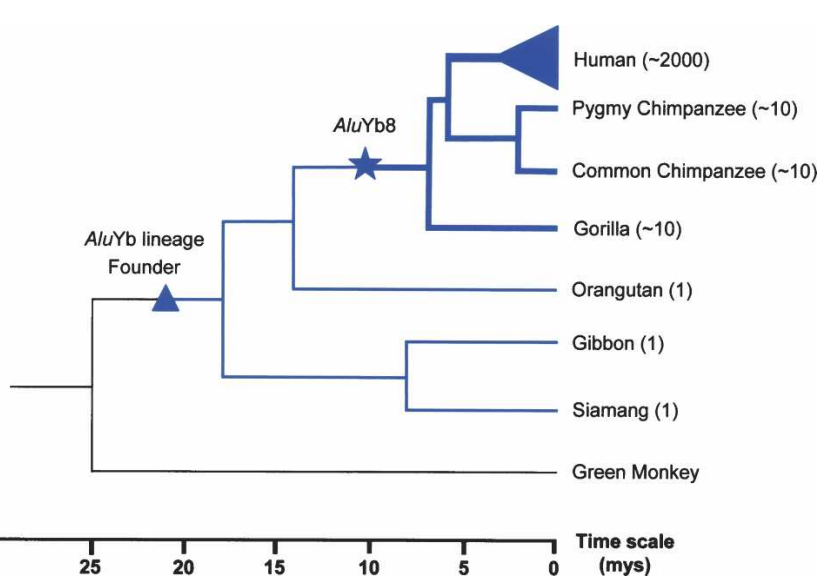

Figure 6. Putative evolutionary scenario for the AluYb lineage. A schematic diagram of the hominid primates is shown with the approximate time scale shown on the bottom in million years. The blue lines indicate the expansion of the AluYb lineage, and the thickness of the lines represents its relative retrotransposition activity. The estimated copy number of AluYb elements in various primates is shown after their names. The blue triangle represents the estimated integration time period of AluYb founder gene, and the star represents the estimated integration time period of the oldest known AluYb8 element (Pan4). 
that some varieties of mobile elements evolve strategies to attenuate their own activity. In Alu, the acquisition of a second monomer to form its dimeric structure has been linked to decreased retrotranspositional activity (Li and Schmid 2004). In vitro modifications to currently active L1 elements can produce orders of magnitude increases in the L1 amplification rate (Han and Boeke 2004). In addition, cryptic polyadenylation sites throughout the L1 sequence may serve to quell the number of full-length, retrotransposition-competent L1 copies generated (Perepelitsa-Belancio and Deininger 2003). Taken together, this evidence suggests that the stealth-driver model may not be a unique feature of the Alu family itself, but rather may be one variant of a common survival strategy for SINE and LINE elements. More generally, the ability of mobile elements to maintain low to moderate levels of amplification activity, rather than more rapid duplication rates, may be a common feature of longlived, successful families of transposons.

\section{Methods}

\section{Computational identification of $A l u Y b$ elements}

A 31-bp (TGCGCCACTGCAGTCCGCAGTCCGGCCTGGG) oligonucleotide that included the $A l u \mathrm{Yb}$ lineage-specific duplication was used to screen the common chimpanzee genome draft sequences (panTro1 Nov. 2003 assembly, http://www.ncbi. nlm.nih.gov; R. Waterston, pers. comm.) using the Basic Local Alignment Search Tool (BLAST) program available at http:// www.ensembl.org/multi/blastview (Altschul et al. 1990). All Alu elements that have the diagnostic 7-bp duplication were selected and extracted along with 1000 bp of unique DNA sequence adjacent to both ends of the elements. The program RepeatMasker (http://repeatmasker.genome.washington.edu/cgi-bin/ RepeatMasker) was then used to annotate all known repeat elements within the DNA sequence. Flanking oligonucleotide primers for the PCR amplification of each Alu element were then designed using Primer3 (http://www-genome.wi.mit.edu/cgibin/primer/primer3_www.cgi). The primers were subsequently screened against the GenBank NR database using BLAST queries to determine whether they resided in unique DNA sequences and would only amplify the Alu elements of interest.

\section{Identification of AluYb elements with PCR display}

The Alu element PCR display methodology has been reported previously (Ray et al. 2005). Using this approach, $500 \mathrm{ng}$ of genomic DNA was partially digested using restriction endonucleases NdeI or MseI as recommended by the manufacturer (New England Biolabs) in $120 \mu \mathrm{L}$ reactions. Digestion products were then ligated with double-stranded linkers and amplified and "displayed" using the primer LNP (5'-GAATTCGTCAACATAG CATTTCT- $3^{\prime}$ ) and an AluYb-specific primer (5'-GGCCGGA CTGCGGACT-3') to acquire partial Alu sequences and the accompanying flanking unique sequences from each template. Since the Alu sequence in the amplicon is about $300 \mathrm{bp}$ long, the PCR products were then purified by BD CHROMAS SPIN-400 columns (BD Biosciences) to select the fragments larger than $400 \mathrm{bp}$, so that enough unique flanking sequence can be obtained to locate the orthologous sequences in the draft sequence of the human genome. A second-round amplification was performed using the LNP oligonucleotide and a second nested AluYbspecific primer (5'-AATCTCGGCTCACTGCAAGCTCCGCT- $\left.3^{\prime}\right)$ to increase the specificity of the amplicons. The second-round PCR products were separated on a $2 \%$ agarose gel, and fragments larger than 400 bp were excised and extracted from the gel using the Wizard gel-purification kit (Promega). The purified products were then cloned into the TOPO-TA cloning vector (Invitrogen). At least 72 clones were randomly isolated from each template, and DNA sequences were determined from both strands using chain-termination sequencing (Sanger et al. 1977) on an ABI 3100 automated DNA sequencer.

After obtaining the sequences, the BLAST-Like Alignment Tool (BLAT) program (http://genome.ucsc.edu/cgi-bin/ hgBlat?command=start) (Kent 2002) was used to compare the resulting partial $A l u \mathrm{Yb} 8$ element and its adjacent flanking sequence with human draft sequences to identify orthologous sequences. Using a combination of the unique flanking sequence and the orthologous human DNA sequences, oligonucleotide primers were designed around each newly identified $A l u \mathrm{Yb}$ element as outlined above.

\section{PCR analysis of $A / u Y b$ elements}

All of the AluYb loci were screened on a panel composed of human genomic DNA (HeLa cell line ATCC CCL-2) and DNA samples from the following nonhuman primate species: Pan troglodytes (common chimpanzee), Pan paniscus (bonobo or pygmy chimpanzee), Gorilla gorilla (lowland gorilla), Pongo pygmaeus (orangutan), Hylobates syndactylus (siamang), Hylobates lar (white handed gibbon), Chlorocebus aethiops sabaeus (green monkey), and Aotus trivirgatus (owl monkey). The nonhuman primate DNA is available as a primate phylogenetic panel (PRP00001) from the Coriell Institute for Medical Research. The chimpanzee-specific $A l u \mathrm{Yb}$ loci were also screened for insertion presence/absence using a common chimpanzee population panel composed of 12 unrelated individuals of unknown geographic origin, which was provided by the Southwest Foundation for Biomedical Research.

PCR amplification of each locus was performed in $25-\mu \mathrm{L}$ reactions using 10-50 ng of target DNA, $200 \mathrm{nM}$ of each oligonucleotide primer, $200 \mu \mathrm{M}$ dNTP's in $50 \mathrm{mM} \mathrm{KCl}, 1.5 \mathrm{mM} \mathrm{MgCl}_{2}, 10 \mathrm{mM}$ Tris- $\mathrm{HCl}$ (pH 8.4), and 2.5 units Taq DNA polymerase. Each sample was subjected to an initial denaturation step of $94^{\circ} \mathrm{C}$ for $150 \mathrm{sec}$, followed by 32 cycles of $1 \mathrm{~min}$ of denaturation at $94^{\circ} \mathrm{C}, 1 \mathrm{~min}$ of annealing at optimal annealing temperature, $1 \mathrm{~min}$ of extension at $72^{\circ} \mathrm{C}$, followed by a final extension step at $72^{\circ} \mathrm{C}$ for $10 \mathrm{~min}$. Resulting PCR products were fractionated on a $2 \%$ agarose gel with 0.25 $\mu \mathrm{g}$ of ethidium bromide and visualized using UV fluorescence.

\section{DNA sequence analysis}

To confirm the presence of AluYb elements, all PCR products suggesting the presence of an Alu element were gel purified using the Wizard gel-purification kit (Promega). Purified PCR products were then cloned into vectors using the TOPO TA cloning kit (Invitrogen) and sequenced using chain-termination sequencing (Sanger et al. 1977) on an Applied Biosystems 3100 automated DNA sequencer. All clones were sequenced in both directions to confirm the sequence. The DNA sequences generated in this study are available in the GenBank under accession numbers AY791249-AY791290.

\section{Acknowledgments}

We thank Dr. David Ray for critical reading and suggestions during the preparation of this manuscript. We also thank Anthony Carter for providing the human AluYb sequences. This research was supported by the Louisiana Board of Regents Millennium Trust Health Excellence Fund HEF (2000-05)-01 (M.A.B.), Na-

\section{Genome Research}

www.genome.org 
tional Science Foundation BCS-0218338 (M.A.B.) and EPS0346411 (M.A.B.), National Institutes of Health RO1 GM59290 (M.A.B.), and the State of Louisiana Board of Regents Support Fund (M.A.B.).

\section{References}

Altschul, S.F., Gish, W., Miller, W., Myers, E.W., and Lipman, D.J. 1990. Basic local alignment search tool. J. Mol. Biol. 215: 403-410.

Bandelt, H.J., Forster, P., and Rohl, A. 1999. Median-joining networks for inferring intraspecific phylogenies. Mol. Biol. Evol. 16: 37-48.

Batzer, M.A. and Deininger, P.L. 2002. Alu repeats and human genomic diversity. Nat. Rev. Genet. 3: 370-379.

Batzer, M.A., Rubin, C.M., Hellmann-Blumberg, U., Alegria-Hartman, M., Leeflang, E.P., Stern, J.D., Bazan, H.A., Shaikh, T.H., Deininger, P.L., and Schmid, C.W. 1995. Dispersion and insertion polymorphism in two small subfamilies of recently amplified human Alu repeats. J. Mol. Biol. 247: 418-427.

Batzer, M.A., Deininger, P.L., Hellmann-Blumberg, U., Jurka, J., Labuda, D., Rubin, C.M., Schmid, C.W., Zietkiewicz, E., and Zuckerkandl, E. 1996. Standardized nomenclature for Alu repeats. J. Mol. Evol. 42: $3-6$.

Britten, R.J. 1994. Evidence that most human Alu sequences were inserted in a process that ceased about 30 million years ago. Proc. Natl. Acad. Sci. 91: 6148-6150.

Britten, R.J., Baron, W.F., Stout, D.B., and Davidson, E.H. 1988. Sources and evolution of human Alu repeated sequences. Proc. Natl. Acad. Sci. 85: 4770-4774.

Carroll, M.L., Roy-Engel, A.M., Nguyen, S.V., Salem, A.H., Vogel, E. Vincent, B., Myers, J., Ahmad, Z., Nguyen, L., Sammarco, M., et al. 2001. Large-scale analysis of the Alu Ya5 and Yb8 subfamilies and their contribution to human genomic diversity. J. Mol. Biol. 311: $17-40$.

Carter, A.B., Salem, A.H., Hedges, D.J., Keegan, C.N., Kimball, B., Walker, J.A., Watkins, W.S., Jorde, L.B., and Batzer, M.A. 2004. Genome-wide analysis of the human Alu Yb-lineage. Hum. Genomics 1: $167-178$.

Cordaux, R., Hedges, D.J., and Batzer, M.A. 2004. Retrotransposition of Alu elements: How many sources? Trends Genet. 20: 464-467.

Deininger, P.L. and Batzer, M.A. 1993. Evolution of retroposons. In Evolutionary biology (eds. M.K. Hecht, et al.), pp. 157-196. Plenum Press, New York.

Deininger, P.L. and Slagel, V.K. 1988. Recently amplified Alu family members share a common parental Alu sequence. Mol. Cell. Biol. 8: $4566-4569$.

Deininger, P.L., Batzer, M.A., Hutchison III, C.A., and Edgell, M.H. 1992. Master genes in mammalian repetitive DNA amplification. Trends Genet. 8: 307-311.

Dewannieux, M., Esnault, C., and Heidmann, T. 2003. LINE-mediated retrotransposition of marked Alu sequences. Nat. Genet. 35: 41-48.

Gibbons, R., Dugaiczyk, L.J., Girke, T., Duistermars, B., Zielinski, R., and Dugaiczyk, A. 2004. Distinguishing humans from great apes with AluYb8 repeats. J. Mol. Biol. 339: 721-729.

Goodman, M., Porter, C.A., Czelusniak, J., Page, S.L., Schneider, H., Shoshani, J., Gunnell, G., and Groves, C.P. 1998. Toward a phylogenetic classification of Primates based on DNA evidence complemented by fossil evidence. Mol. Phylogenet. Evol. 9: 585-598.

Han, J.S. and Boeke, J.D. 2004. A highly active synthetic mammalian retrotransposon. Nature 429: 314-318.

Hedges, D.J., Callinan, P.A., Cordaux, R., Xing, J., Barnes, E., and Batzer, M.A. 2004. Differential Alu mobilization and polymorphism among the human and chimpanzee lineages. Genome Res. 14: 1068-1075.

Jurka, J. 1993. A new subfamily of recently retroposed human Alu repeats. Nucleic Acids Res. 21: 2252.

Jurka, J. and Smith, T. 1988. A fundamental division in the Alu family of repeated sequences. Proc. Natl. Acad. Sci. 85: 4775-4778.

Jurka, J., Krnjajic, M., Kapitonov, V.V., Stenger, J.E., and Kokhanyy, O. 2002. Active $A l u$ elements are passed primarily through paternal germlines. Theor. Popul. Biol. 61: 519-530.

Kass, D.H., Batzer, M.A., and Deininger, P.L. 1995. Gene conversion as a secondary mechanism of short interspersed element (SINE) evolution. Mol. Cell. Biol. 15: 19-25.

Kent, W.J. 2002. BLAT-the BLAST-like alignment tool. Genome Res. 12: $656-664$.

Kim, J., Martignetti, J.A., Shen, M.R., Brosius, J., and Deininger, P. 1994. Rodent BC1 RNA gene as a master gene for ID element amplification. Proc. Natl. Acad. Sci. 91: 3607-3611.

Lander, E.S., Linton, L.M. Birren, B., Nusbaum, C., Zody, M.C., Baldwin,
J., Devon, K., Dewar, K., Doyle, M., FitzHugh, W., et al. 2001. Initial sequencing and analysis of the human genome. Nature

409: 860-921.

Leeflang, E.P., Liu, W.M., Chesnokov, I.N., and Schmid, C.W. 1993. Phylogenetic isolation of a human Alu founder gene: Drift to new subfamily identity [corrected]. J. Mol. Evol. 37: 559-565.

Li, T.H. and Schmid, C.W. 2004. Alu's dimeric consensus sequence destabilizes its transcripts. Gene 324: 191-200.

Maeda, N., Wu, C.I., Bliska, J., and Reneke, J. 1988. Molecular evolution of intergenic DNA in higher primates: Pattern of DNA changes, molecular clock, and evolution of repetitive sequences. Mol. Biol. Evol. 5: 1-20.

Matera, A.G., Hellmann, U., Hintz, M.F., and Schmid, C.W. 1990. Recently transposed $A l u$ repeats result from multiple source genes. Nucleic Acids Res. 18: 6019-6023.

Muratani, K., Hada, T., Yamamoto, Y., Kaneko, T., Shigeto, Y., Ohue, T., Furuyama, J., and Higashino, K. 1991. Inactivation of the cholinesterase gene by Alu insertion: Possible mechanism for human gene transposition. Proc. Natl. Acad. Sci. 88: 11315-11319.

Oldridge, M., Zackai, E.H., McDonald-McGinn, D.M., Iseki, S., Morriss-Kay, G.M., Twigg, S.R., Johnson, D., Wall, S.A., Jiang, W. Theda, C., et al. 1999. De novo Alu-element insertions in FGFR2 identify a distinct pathological basis for Apert syndrome. Am. J. Hum. Genet. 64: 446-461.

Otieno, A.C., Carter, A.B., Hedges, D.J., Walker, J.A., Ray, D.A., Garber, R.K., Anders, B.A., Stoilova, N., Laborde, M.E., Fowlkes, J.D., et al. 2004. Analysis of the human Alu Ya-lineage. J. Mol. Biol. 342: 109-118.

Pascale, E., Liu, C., Valle, E., Usdin, K., and Furano, A.V. 1993. The evolution of long interspersed repeated DNA (L1, LINE 1) as revealed by the analysis of an ancient rodent L1 DNA family. J. Mol. Evol. 36: 9-20.

Perepelitsa-Belancio, V. and Deininger, P. 2003. RNA truncation by premature polyadenylation attenuates human mobile element activity. Nat. Genet. 35: 363-366.

Posada, D. and Crandall, K.A. 2001. Intraspecific gene genealogies: Trees grafting into networks. Trends Ecol. Evol. 16: 37-45.

Price, A.L., Eskin, E., and Pevzner, P.A. 2004. Whole-genome analysis of Alu repeat elements reveals complex evolutionary history. Genome Res. 14: 2245-2252.

Ray, D.A., Xing, J.C., Hedges, D.J., Hall, M.A., Laborde, M.E., Anders, B.A., White, B.R., Stoilova, N., Fowlkes, J.D., Landry, K.E., et al. 2005. Alu insertion loci and platyrrhine primate phylogeny. Mol. Phylogenet. Evol. (in press).

Roos, C., Schmitz, J., and Zischler, H. 2004. Primate jumping genes elucidate strepsirrhine phylogeny. Proc. Natl. Acad. Sci. 101: $10650-10654$

Roy-Engel, A.M., Carroll, M.L., Vogel, E., Garber, R.K., Nguyen, S.V., Salem, A.H., Batzer, M.A., and Deininger, P.L. 2001. Alu insertion polymorphisms for the study of human genomic diversity. Genetics 159: $279-290$

Roy-Engel, A.M., Carroll, M.L., El-Sawy, M., Salem, A.H., Garber, R.K., Nguyen, S.V., Deininger, P.L., and Batzer, M.A. 2002.

Non-traditional Alu evolution and primate genomic diversity. J. Mol. Biol. 316: 1033-1040.

Salem, A.H., Kilroy, G.E., Watkins, W.S., Jorde, L.B., and Batzer, M.A. 2003a. Recently integrated Alu elements and human genomic diversity. Mol. Biol. Evol. 20: 1349-1361.

Salem, A.H., Ray, D.A., Xing, J.C., Callinan, P.A., Myers, J.S., Hedges, D.J., Garber, R.K., Witherspoon, D.J., Jorde, L.B., and Batzer, M.A 2003b. Alu elements and hominid phylogenetics. Proc. Natl. Acad. Sci. 100: 12787-12791.

Sanger, F., Nicklen, S., and Coulson, A.R. 1977. DNA sequencing with chain-terminating inhibitors. Proc. Natl. Acad. Sci. 74: 5463-5467.

Schmid, C.W. 1993. How many source Alus? Trends Genet. 9: 39.

Shaikh, T.H. and Deininger, P.L. 1996. The role and amplification of the HS Alu subfamily founder gene. J. Mol. Evol. 42: 15-21.

Shen, M.R., Batzer, M.A., and Deininger, P.L. 1991. Evolution of the master Alu gene(s). J. Mol. Evol. 33: 311-320.

Singer, S.S., Schmitz, J., Schwiegk, C., and Zischler, H. 2003. Molecular cladistic markers in New World monkey phylogeny (Platyrrhini, Primates). Mol. Phylogenet. Evol. 26: 490-501.

Slagel, V., Flemington, E., Traina-Dorge, V., Bradshaw, H., and Deininger, P. 1987. Clustering and subfamily relationships of the Alu family in the human genome. Mol. Biol. Evol. 4: 19-29.

Willard, C., Nguyen, H.T., and Schmid, C.W. 1987. Existence of at least three distinct Alu subfamilies. J. Mol. Evol. 26: 180-186.

Xing, J.C., Salem, A.H., Hedges, D.J., Kilroy, G.E., Watkins, W.S., Schienman, J.E., Stewart, C.B., Jurka, J., Jorde, L.B., and Batzer, M.A. 2003. Comprehensive analysis of two Alu Yd subfamilies. J. Mol. Evol. 57: S76-S89. 


\section{Han et al.}

Xing, J.C., Hedges, D.J., Han, K., Wang, H., Cordaux, R., and Batzer, M.A. 2004. Alu element mutation spectra: Molecular clocks and the effect of DNA methylation. J. Mol. Biol. 344: 675-682.

Zietkiewicz, E., Richer, C., Makalowski, W., Jurka, J., and Labuda, D. 1994. A young Alu subfamily amplified independently in human and African great apes lineages. Nucleic Acids Res. 22: 5608-5612.

\section{Web site references}

http://batzerlab.lsu.edu; Batzer laboratory.

http://www.ensembl.org/multi/blastview; Basic Local Alignment Search
Tool (BLAST).

http://www.fluxus-engineering.com/sharenet.htm; NETWORK 4.1. http://repeatmasker.genome.washington.edu/cgi-bin/RepeatMasker; RepeatMasker.

http://www-genome.wi.mit.edu/cgi-bin/primer/primer3_www.cgi; Primer3.

http://genome.ucsc.edu/cgi-bin/hgBlat?command=start; BLAT.

Received November 19, 2004; accepted in revised form January 28, 2005. 


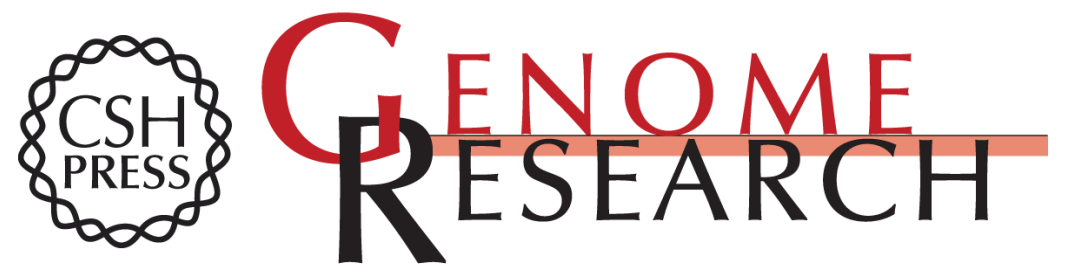

\section{Under the genomic radar: The Stealth model of Alu amplification}

Kyudong Han, Jinchuan Xing, Hui Wang, et al.

Genome Res. 2005 15: 655-664

Access the most recent version at doi:10.1101/gr.3492605

References This article cites 49 articles, 14 of which can be accessed free at:

http://genome.cshlp.org/content/15/5/655.full.html\#ref-list-1

\section{License}

Email Alerting Receive free email alerts when new articles cite this article - sign up in the box at the Service top right corner of the article or click here.

\section{Affordable, Accurate Sequencing.}

To subscribe to Genome Research go to: https://genome.cshlp.org/subscriptions 